\title{
ATLAS B0 Toroid Model Coil Test at CERN
}

\author{
Paola Miele, E. Acerbi, R. Berthier, H. Boxman, F. Broggi, F. Cataneo, A. Dael, N. Dolgetta, A. Dudarev, A. Foussat, \\ F. Haug, H. ten Kate, C. Mayri, A. Paccalini, G. Rivoltella, L. Rossi, E. Sbrissa, H. Tyrvainen, and G. Volpini
}

\begin{abstract}
The ATLAS superconducting magnet system consists of a Barrel Toroid, two End-Cap Toroids and a Central Solenoid. The Barrel Toroid, with overall dimensions of 20-m diameter by 26-m length, is made of eight individual coils symmetrically assembled around the central axis with a warm structure. The system is presently under construction in industry. In order to verify the construction concepts a model coil $\mathrm{BO}$, a 9-m short version of a single Barrel Toroid coil, was built. Since April 2001, an extensive test program is underway at CERN to characterize the mechanical, thermal, electrical and magnetic properties of the coil. The magnet successfully achieved the 20-kA nominal operating current in July 2001. The test program and the main results are reported.
\end{abstract}

Index Terms-Magnets, superconductors, test facility, toroids.

\section{INTRODUCTION}

$\mathbf{T}$ HE ATLAS Magnet System [1]-[3] comprises the superconducting magnets and the related services, such as power supply, cryogenic, vacuum and control and safety systems. The magnet system has overall dimensions of 26 meters in length and 20 meters in diameter, Fig. 1. The magnet system consists of a Barrel Toroid (BT), two End-Cap Toroids (ECT) and the Central Solenoid (CS). Each of the three toroids consists of eight coils connected in series, with flat pancake type of windings, assembled around the beam axis with an offset angle of $22.5^{\circ}$ between the BT and ECT systems, in order to optimize the bending power in the available space. A model coil of the Barrel Toroid of reduced length, the B0 model coil, has been constructed to verify design concepts as well as to exercise the manufacturing techniques. The coils are built with Aluminum stabilized $\mathrm{NbTi} / \mathrm{Cu}$ superconductor, insulated with glass tape and fully impregnated with epoxy resin. Indirect cooling at $4.5 \mathrm{~K}$ by forced flow of liquid Helium is adopted. The electrical system consists of a $21-\mathrm{kA}$ common TF power system and an 8-kA CS power system and related quench protection systems.

A facility was built at CERN for testing first the B0 model coil and thereafter all other coils before installation. The facility includes a He refrigerator with cold $\mathrm{He}$ pumps providing forced Helium flow up to $100 \mathrm{~g} / \mathrm{s}$ for the cold mass and thermal shield cooling at $4.5 \mathrm{~K}$ and $60 \mathrm{~K}$, respectively, a $6 \mathrm{~V} / 24 \mathrm{kA}$ electrical system, the vacuum system providing $10^{-4} \mathrm{mbar}$, the quench

Manuscript received September 24, 2001. This work was supported by the ATLAS Collaboration.

P. Miele, H. Boxman, F. Cataneo, A. Dudarev, A. Foussat, F. Haug, H. ten Kate, E. Sbrissa, and H. Tyrvainen are with CERN, Geneva, Switzerland (e-mail: Paola.Miele@CERN.ch).

E. Acerbi, F. Broggi, A. Paccalini, G. Rivoltella, L. Rossi, and G. Volpini are with INFN-LASA, Milano, Italy.

R. Berthier, A. Dael, and C. Mayri are with CEA, Saclay, France.

N. Dolgetta is with Euratom-CEA, Cadarache, F.

Publisher Item Identifier S 1051-8223(02)03574-1.

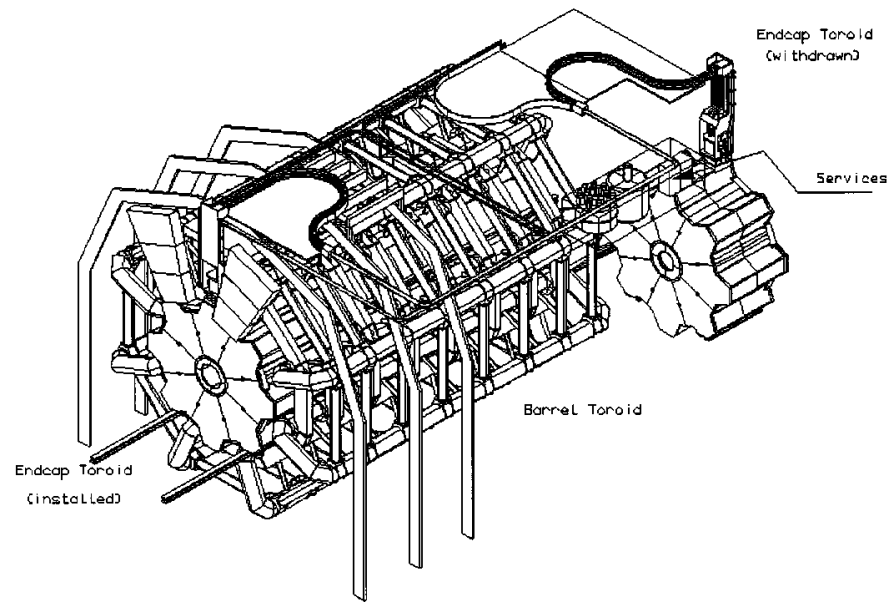

Fig. 1. The ATLAS Magnet System showing the three toroids.

protection, control and diagnostics systems. Because of its exceptional size, it is not feasible to test the fully integrated BT on surface prior to underground installation. Each coil is tested on surface with a magnetic mirror to simulate the force load in the assembled toroid. The test facility was commissioned in autumn 2000, using the model coil B00 [4], []. The B0 test is planned in the year 2001. The test of the eight BT coils is scheduled for the years 2002-2004, with about four months time allocated for each magnet.

\section{MOdel COIL B0}

B0 [5], Fig. 2, is a model of the BT coils with the same design and construction concepts. The width and cross-section of $\mathrm{B} 0$ are the same as for the BT coils, while a reduced length of $9 \mathrm{~m}$ was chosen to reduce cost and test requirements. The cold mass consists of two double pancakes connected in series and housed in a common $\mathrm{Al}$ alloy casing. Two ribs connecting the 2 long girders react the internal magnetic forces. The coil has a stainless steel cylindrical vacuum vessel enclosing the cold mass. The cold mass support system consists of three titanium tie rods reacting the radial electromagnetic forces and the cryogenic stops to center the cold mass in the transverse direction. The fixed point reacting the longitudinal load consists of two rods solidly attached to the central rib of the coil casing and welded to the vacuum vessel. Two cooling circuits (one redundant) provide Helium circulation and cooling of the cold mass to $4.5 \mathrm{~K}$.

Integration of the cold mass in the vacuum vessel, Fig. 3, includes gluing of the cooling pipes onto the cold mass, assembly of the cold mass supports, i.e., tie rods and cryogenic stops, assembly of the Al alloy thermal shields, assembly of the superinsulation and closing of the vacuum vessel by welding. B0 


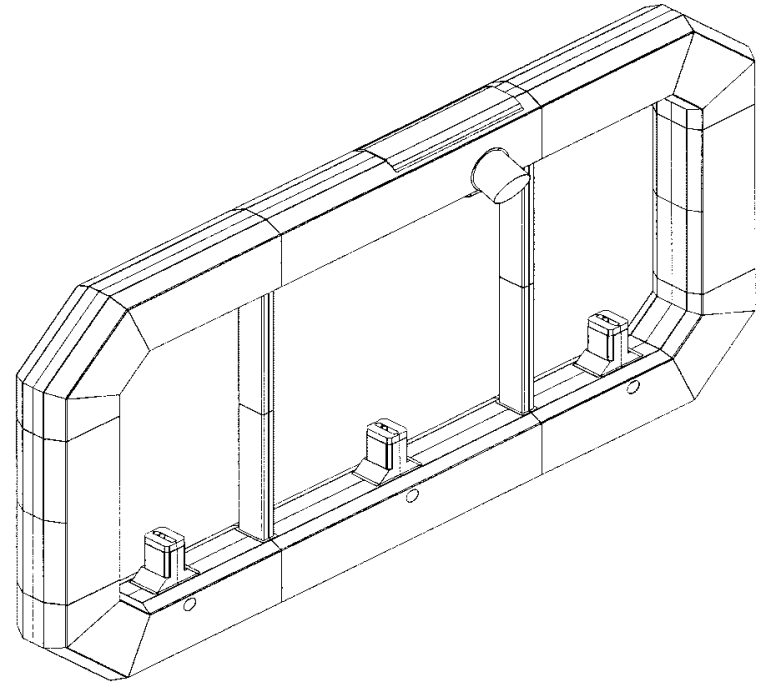

Fig. 2. B0 model coil.

has allowed optimization of the construction methods and procedures, namely the winding and impregnation procedures, the cold mass integration, the cryogenic stop sliding mechanism and the vacuum vessel integration scenario and procedure [6]. The main parameters for BT and B0 are listed in Table I.

\section{A. Instrumentation}

Sensors and actuators are implemented, such as temperature sensors, voltage taps, pick-up coils, protection quench heaters, point heaters, strain gauges, load cells, hall probes in the joints, Superconducting Quench Detectors (SQD), Rogowsky coils and positioning sensors. The voltage taps for quench detection are placed at the current leads, the internal double pancake junctions and the double pancake inlets/outlets. Four quench heaters allow for triggering the quench. Voltage taps for propagation studies are distributed around the point heaters. For measuring quench propagation velocity pick-up coils are installed. The thermal map during cool down and operation is derived with carbon sensors, CLTS, Pt resistors, and thermocouples.

\section{B. Test Setup}

The test of B0 with magnetic mirror, Fig. 4, is performed in horizontal position. Turn-over and handling tests without magnetic mirror are planned. Two turrets are used for the connections to the external services. The Current Lead Turret (CLT) at the top contains the connections to the bus bars via 21-kA current leads. The Cryogenic Service Turret (CST) at the bottom houses the connections to the cryogenic transfer lines and the junction of the two double pancakes. The B0 test program comprises two phases, i.e., commissioning of the coil and dedicated measurements.

Commissioning includes:

- Cool down to $4.5 \mathrm{~K}$, current cycling at various levels up to the nominal current of $20.5 \mathrm{kA}$ and warm up to room temperature;

- In the preceding phases verification of the mechanical, magnetic, thermal and electrical behavior of the coil;

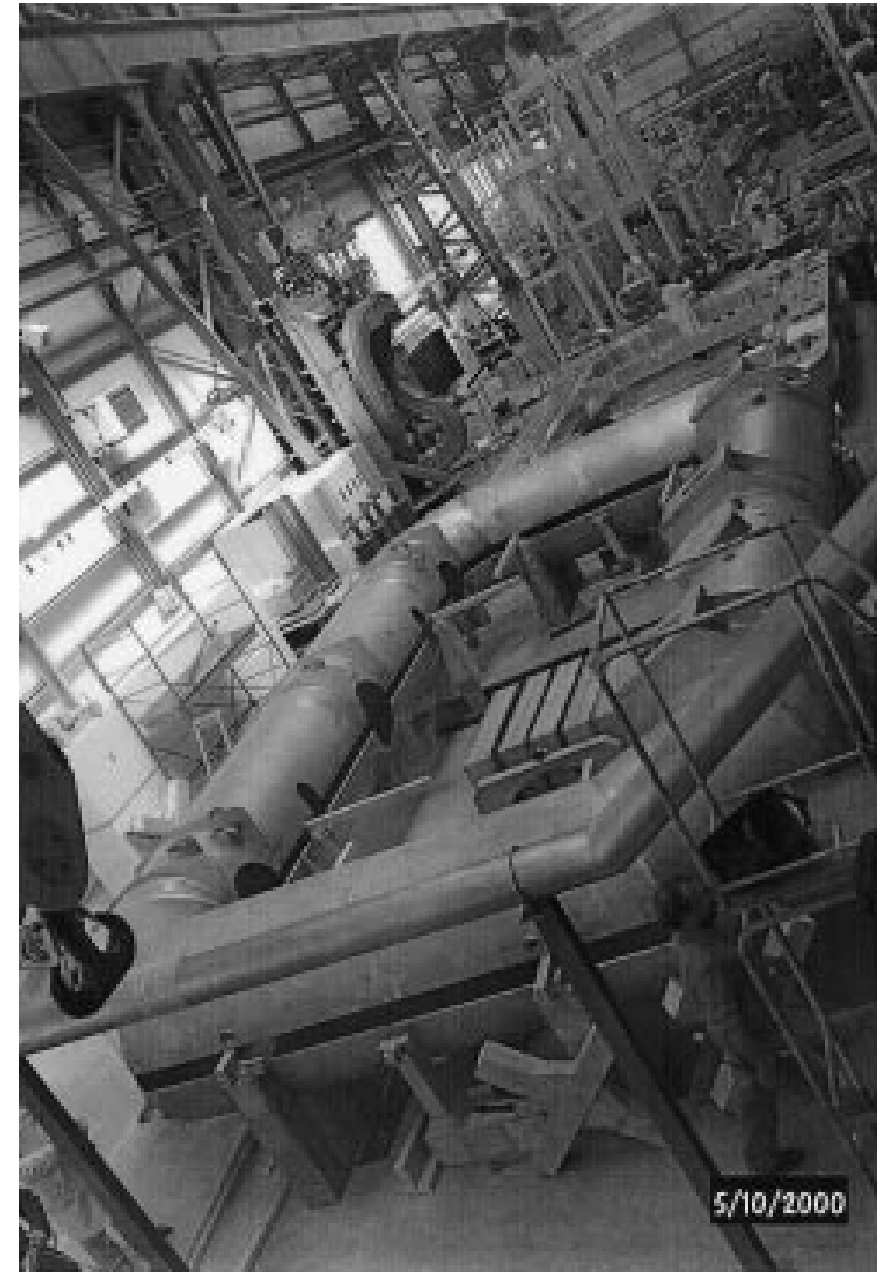

Fig. 3. Integration of the B0 cold mass in the vacuum vessel.

TABLE I

B0 AND BT COIL MAIN PARAMETERS

\begin{tabular}{lll}
\hline \hline & B0 & BT \\
Number of coils & 1 & 8 \\
Racetrack Shape $[\mathrm{m} \mathrm{x} \mathrm{m}]$ & $5 \times 9$ & $5 \times 26$ \\
${\text { Cable Cross Section }\left[\mathrm{mm}^{2}\right]}^{\text {Operating Current [kA] }}$ & $57 \times 12$ & \\
Stored Energy [MJ] & 20.5 & 1080 \\
Operating Temperature [K] & 46 & \\
Critical Current $(@ 4.2 \mathrm{~K}, 5 \mathrm{~T})[\mathrm{kA}]$ & 4.5 & \\
Maximum Magnetic Field [T] & $>47.5$ & \\
RRR Al & 4 & 1300 \\
Force to Magnetic Mirror [t] & $>1200$ & 832 \\
Total weight [t] & 400 &
\end{tabular}

- Verification of the cryogenic performance;

- Verification of the current ramp;

- Test of quench heaters for protection of the coil;

- Verification of the fast and slow discharge behavior.

Dedicated measurements include:

- Verification of heat losses and thermal map;

- Current lead and resistive junction performance;

- Details of electromagnetic and thermal stability;

- Normal zone and quench propagation;

- Redundant cooling circuit test;

- Test at current higher than the nominal value; 


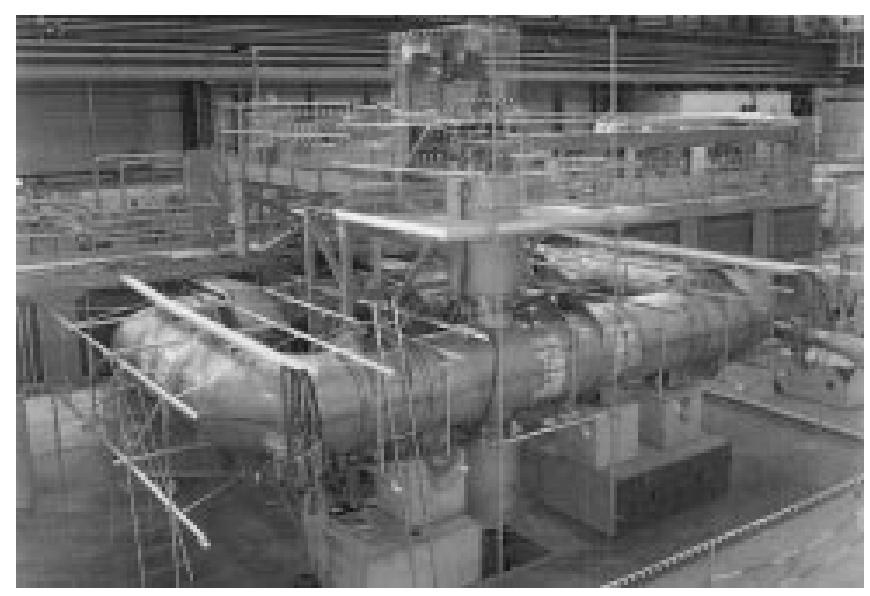

Fig. 4. Test of B0 with magnetic mirror at CERN.

- Magnetic field measurements.

\section{TEST FACILTY}

The integration and testing of the coils is performed in Hall 180 at CERN [7]. The test station comprises two test benches separated by a magnetic mirror and coil support structures, cryoplant, power supply, vacuum pumps, Magnet Control System (MCS) and Magnet Safety System (MSS) as well as control rooms and workshops. Early 2001 the B00 model coil has been successfully tested with the aim to commission the test station and to study the three different ATLAS superconductors in operating conditions to study ramp losses, stability and quench propagation. The test equipment will also be used for the test of the two ECT magnets fully assembled.

\section{A. Cryogenic System}

The cryogenic system [8], comprises the refrigerator cold box providing $1.2 \mathrm{~kW}$ at $4.5 \mathrm{~K}$ and related compressor, the pump cryostat containing two immersed centrifugal pumps, distribution valve box, liquid nitrogen precooling unit, three transfer lines feeding the test benches, as well as instrumentation and process controls. The pump circuit is independent from the refrigerator allowing for safe ramp down of the coil in case of failure of the refrigerator, with $2 \mathrm{~h}$ of autonomy.

\section{B. Power Supply System}

The system comprises a 6-V/24-kA switch mode power converter and DCCT for reading the current in the coil, $230 \mathrm{VAC}$ UPS that guarantees the magnet safety, a $0.3-\div 1-\mathrm{m} \Omega$ diode/resistor dump unit, electromechanical circuit breaker, 80-m long $1-\mu \Omega / \mathrm{m}$ aluminum bus bars connected to the current leads in the CLT turrets.

\section{Magnet Control and Safety Systems}

The protection of the coils is based on the external dump of the stored energy through the use of a dump resistor unit of low value in the case of slow dump and the initiation of quenches in all coils by means of protection quench heaters in the fast dump event. In slow and fast discharge modes the stored energy of about $46 \mathrm{MJ}$ for B0 and $113 \mathrm{MJ}$ for a single BT coil

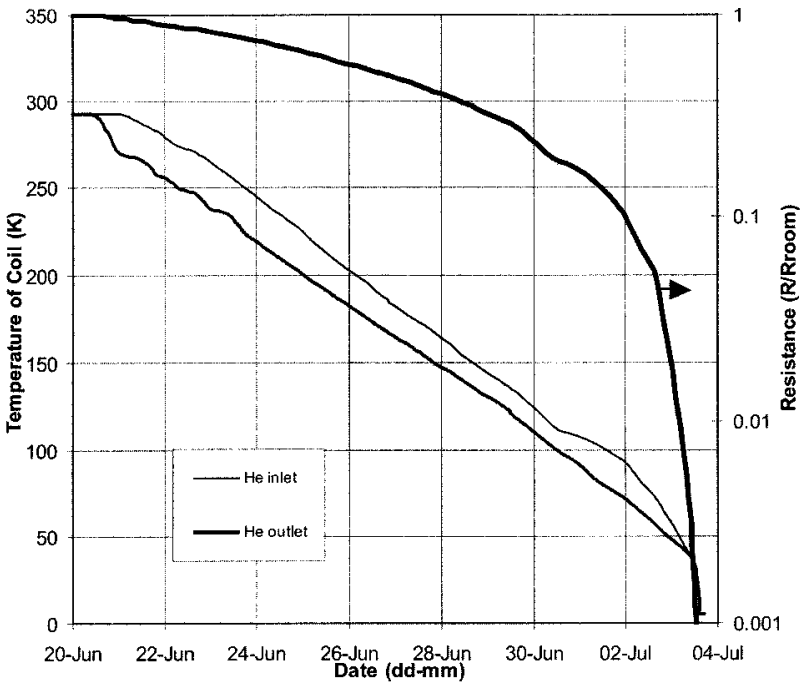

Fig. 5. Cool down phase to $4.5 \mathrm{~K}$.

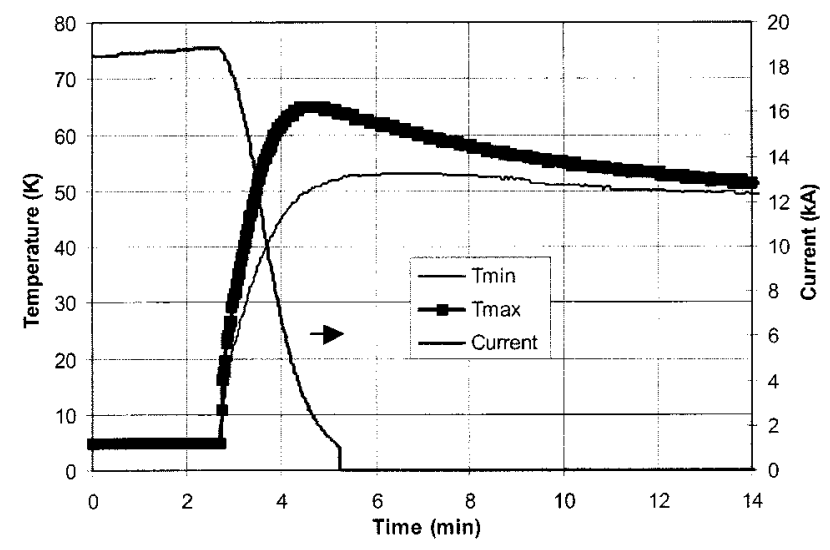

Fig. 6. Temperature increase due to fast dump at $19 \mathrm{kA}$.

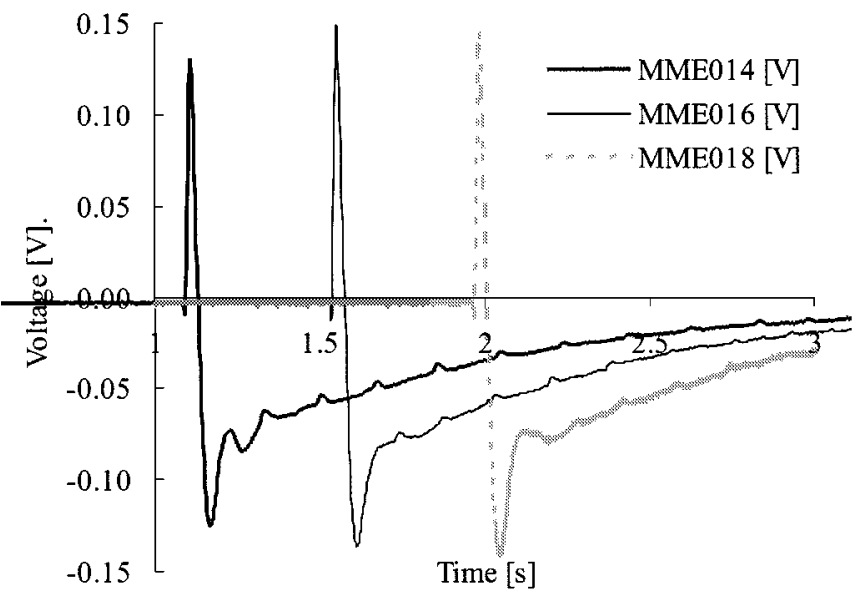

Fig. 7. Pick up coil signals showing flux changing.

is dissipated respectively in the dump unit and in the coil, with time constants of about $2400 \mathrm{~s}$ and $80 \mathrm{~s}$ in the two cases. Dual redundancy is implemented on safety sensors and channels. A new Superconductor Quench Detector (SQD) detects a quench in the bus bar with high resolution. The protection of the magnet relies on eight temperature sensors in the superconducting bus lines and current leads, three SQDs and three bridge detection 


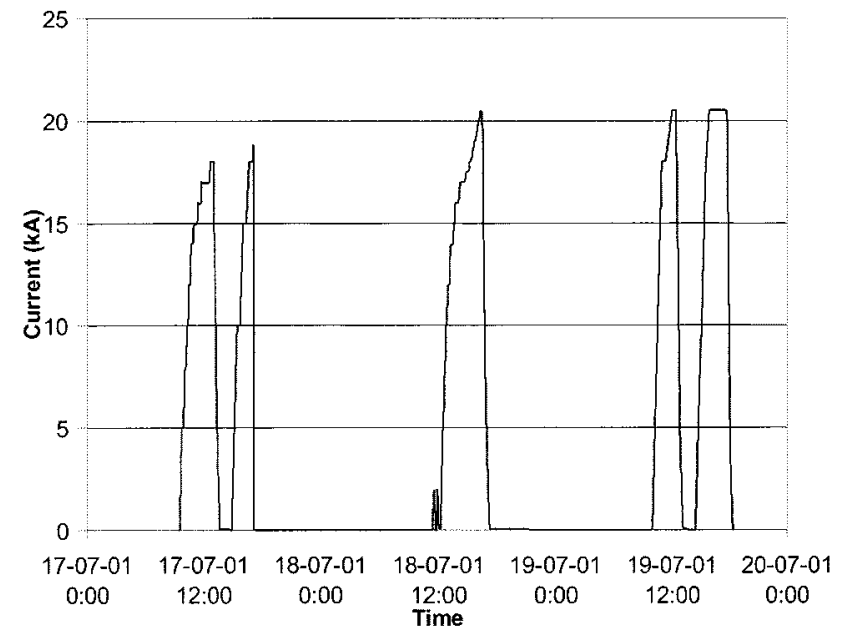

Fig. 8. Various ramp up, slow dump cycles to 0-20.5-0 kA.

systems based on unbalanced voltage readings through the pancakes. MCS provides regulation and monitoring of all the functional parameters of the magnet. It consists of sensors and actuators, PLCs, the supervisor system, and fast and slow Data Acquisition Systems.

\section{TEST RESUlts}

The first B0 test campaign has proven the successful operation of the coil in its complete cycle, i.e., cool down, current up to nominal current $I=20.5 \mathrm{kA}$ and warm up.

\section{A. Cool Down Phase}

Cool down from $300 \mathrm{~K}$ to $4.5 \mathrm{~K}$ is performed with automatic control of the maximum differential temperature at $40 \mathrm{~K}$, to limit the induced stress. The time required to reach $4.5 \mathrm{~K}$ is two weeks. Fig. 5 shows the temperature at the Helium inlet/outlet and the Aluminum resistance drop, which confirms the specified RRR of more than 1200. A homogenous distribution of the temperature is observed in the coil. The mechanical behavior of the coil must be monitored during cool down, to verify soundness of the support system. The cryogenic stops allow for the thermal contraction of the coil up to $37 \mathrm{~mm}$ via a sliding system, while the articulated tie rods allow for rotation via sliding cylindrical surfaces in a preloaded configuration. The mechanical stress in the support system parts has been considerably below the acceptable limit values.

\section{B. Excitation Phase}

Excitation of the coil was performed in steps up to $20.5 \mathrm{kA}$. At the first ramp a quench occurred at about $19 \mathrm{kA}$ in the superconducting bus lines external to the coil. Fig. 6 shows the temperature increase of the coil up to maximum $65 \mathrm{~K}$. About 12 hours was the recovery time of the cryogenic system. Proper quench detection and triggering of the protection heaters was demonstrated. The quench propagation velocity was measured with high precision. Fig. 7 shows the signal from the pick up coils, which detect any magnetic flux variation and therefore allows for a precise measurement of the quench propagation velocity. From the delay of different pick up signals along the coil and

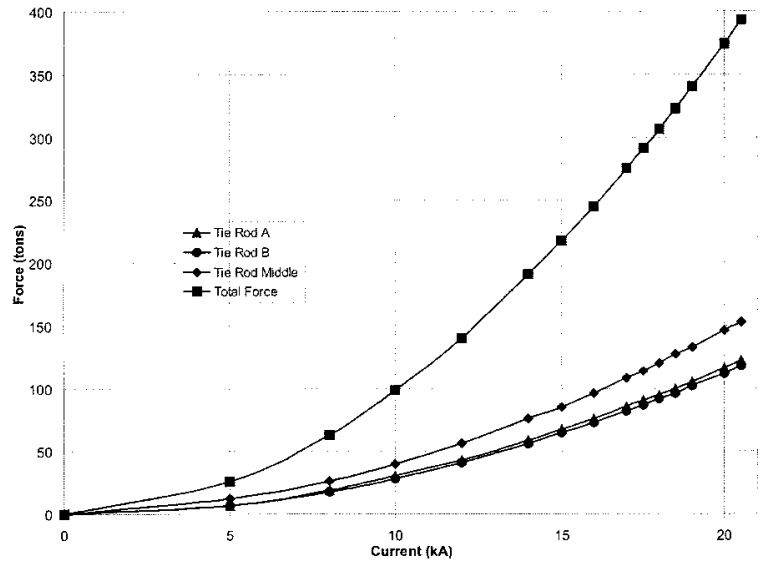

Fig. 9. Forces on tie rods when ramping to $20.5 \mathrm{kA}$.

from two successive peaks in the same signal the longitudinal and transverse quench propagation velocity, respectively, can be deducted. The values found are $v_{/ /} \approx 7 \mathrm{~m} / \mathrm{s}$ and $v_{\perp} \approx 7 \mathrm{~cm} / \mathrm{s}$, respectively. The quench was caused by mechanical movement of the bus bars linking the coil and the current leads. This confirms that the clamping system of the bus lines is a critical item, since it must allow for thermal contraction and rigid support at the same time. After the quench, the nominal current of $20.5 \mathrm{kA}$ was reached many times and maintained, Fig. 8.

The slow discharge process through the dump unit was verified and the behavior of the coil junctions analyzed. At high current rates beyond $100 \mathrm{~A} / \mathrm{s}$ significant induced eddy currents in the internal pancake junction were observed, which nevertheless did not lead to instability. During normal coil operation the ramp rates are much lower, below $4 \mathrm{~A} / \mathrm{s}$. Herewith the junction technique is proven. The mechanical behavior of the coil was verified and is consistent with the calculations [9]. Fig. 9 shows the forces measured in the three tie rods, which gives a total radial force of $400 \mathrm{t}$ complying with the load in the operational BT configuration. The coil deformation is extrapolated from the strain gauge readings on the cold mass.

\section{CONCLUSION}

The test results of the model B0 in general and the ramping up to $20.5 \mathrm{kA}$ in particular, have demonstrated the feasibility of the design and manufacturing techniques of the full size Barrel Toroid. The operational behavior and performance of the coil and related service and control systems have been validated. A next test campaign of the B0 coil is planned in autumn 2001 to investigate further the operational scenarios, safety margins of the systems and the properties of the coil in great detail.

The successful completion of testing the B0 coil is a major step in demonstrating the feasibility and reliability of the ATLAS Magnet System, which for its exceptional size represents a unique challenge in magnet technology.

\section{ACKNOWLEDGMENT}

The authors would like to thank the members of the magnet team at CEA (F), INFN-LASA (I), and CERN for their contributions to this paper. 


\section{REFERENCES}

[1] ATLAS Magnet System Technical Design Report—4 Volumes, , CERN document CERN/LHHC/97-18, 19, 20, 21, 1997.

[2] H. ten Kate, "ATLAS superconducting magnet system status," presented at the Conf. MT-17.

[3] P. Miele and H. ten Kate, "The superconducting magnet system for the ATLAS detector at CERN," SOFT 21, Madrid, Spain, 2000.

[4] A. Dudarev et al., "The B00 model coil for the ATLAS magnet test facility," in Proc. ASC, VA, USA, 2000.
[5] A. Dael et al., "Construction of the ATLAS B0 model coil," in Proc. ASC 2000, vol. 11, VA, USA, 2000, p. 1597.

[6] P. Miele et al., "ATLAS magnet industrial production," presented at the Conf. MT-17.

[7] P. Miele et al., "The ATLAS magnet test facility at CERN," in Proc. ASC 2000, vol. 11, VA, USA, 2000, p. 1713.

[8] F. Haug et al., "The CERN cryogenic test facility for the ATLAS Barrel Toroid magnets," in Proc. MT-16, FL, USA, 1999, p. 1514.

[9] A. Foussat et al., "Mechanical behavior of the ATLAS B0 model coil," presented at the Conf. MT-17. 